Tohoku J. Exp. Med., 2009, 218, 99-105

\title{
Elevation of Serum KL-6 Glycoprotein or Surfactant Protein-D in Adult T-cell Leukemia with Distinct Pulmonary Complications
}

\author{
Akemi Osaka, ${ }^{1}$ Katsunori Yanagihara, ${ }^{1}$ Yasuaki Yamada, ${ }^{1}$ Hiroo Hasegawa, ${ }^{1}$ \\ Naoko Inokuchi, ${ }^{1}$ Tomayoshi Hayashi, ${ }^{1}$ Minori Komoda, ${ }^{2}$ Shigeki Nakamura, ${ }^{3}$ \\ Muneo Aoyama, ${ }^{4}$ Takashi Sawada ${ }^{5}$ and Shimeru Kamihira ${ }^{1}$

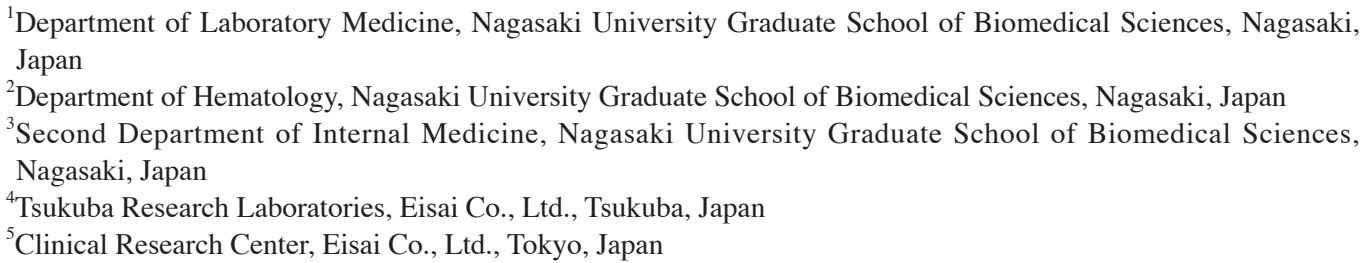

Patients with hematological malignancies frequently suffer from lung diseases as a complication. However, it is difficult to discriminate leukemic invasion into the lung from infectious pulmonary complications. The serum level of Krebs von den Lungen-6 (KL-6), which is a mucin-like glycoprotein, is increased in more than $70 \%$ of patients with interstitial pneumonia. Surfactant protein-D (SP-D) is produced mainly in the lung by alveolar type II and bronchiolar epithelial cells and is a useful serum marker for interstitial pneumonia. We therefore measured the levels of KL-6 and SP-D in sera from 128 patients (76 males and 52 females, mean age: 59 years) with hematological malignancies, including adult T-cell leukemia (ATL). Overall, the increase in KL-6 or SP-D, above each cut-off value $(500 \mathrm{U} / \mathrm{ml}$ for $\mathrm{KL}-6$ and $110 \mathrm{ng} / \mathrm{ml}$ for SP-D), was detected in 11 patients $(8.6 \%)$ or 10 patients $(7.8 \%)$, respectively. In contrast, among 67 ATL patients, 15 patients had high serum levels of KL- 6 and/or SP-D; both were elevated in 2 patients, only KL- 6 was elevated in 6 patients and only SP-D was elevated in 7 patients. Thus, serum KL-6 and SP-D appear to be elevated in a mutually exclusive manner in ATL. Indeed, high serum levels of KL-6 were closely related to the stage of ATL, while the serum SP-D was elevated in ATL patients with pulmonary infection. In conclusion, the combined measurement of KL-6 and SP-D in ATL may become a useful means to discriminate leukemic pulmonary lesions from infectious pulmonary complications. —— Krebs von den Lungen-6; surfactant protein-D; hematological malignancy; adult T-cell leukemia; interstitial pneumonia.

Tohoku J. Exp. Med., 2009, 218 (2), 99-105. (C) 2009 Tohoku University Medical Press

Krebs von den Lungen-6 (KL-6) and surfactant protein-D (SP-D) are sensitive markers of various interstitial pneumonias; however, the use of these markers for patients with hematological malignancies, which are frequently accompanied by lung disorders related to infections and therapies, has not been established (Kohno et al. 1999). KL-6 is a mucin-like glycoprotein that was originally discovered with a murine monoclonal antibody from a hybridoma derived from mouse splenocytes immunized with a human pulmonary adenocarcinoma cell line (Kohno et al. 1988). This KL-6 antibody recognizes a sialylated carbohydrate chain on a high molecular weight glycoprotein, later defined as a muc-1-associated epitope, implying that KL-6 is MUC1 (Stahel et al. 1994). MUC1 is predominantly membrane-bound, consisting of a cytoplasmic tail, a trans- membrane domain and an extracellular domain. Shedding by a metalloproteinase appears to yield a soluble form of MUC1, KL-6, into serum and body fluids in vivo (Marcaurelle and Bertozzi 2002). Indeed, soluble, serum KL-6 has been reported to be elevated in benign lung diseases involving regenerating-pneumocytes that express high levels of MUC1 (Kohno et al. 1988). Serum KL-6 is increased in more than $70 \%$ of patients with interstitial pneumonia (Kohno 1999). Thus, soluble KL-6 has clinically applications as a sensitive and valuable marker for the diagnosis and monitoring of patients with interstitial pneumonia including idiopathic fibrosis, hypersensitive pneumonitis, collagen-related pneumonitis, and radiation pneumonitis. However, serum KL-6 is also elevated in malignant diseases derived from epithelial cells, and is elevated in 30

Received January 9, 2009; revision accepted for publication April 10, 2009.

Correspondence: Katsunori Yanagihara MD, PhD, Department of Laboratory Medicine, Nagasaki University Graduate School of

Biomedical Sciences, 1-7-1, Sakamoto, Nagasaki City, Nagasaki 852-8501, Japan.

e-mail: k-yanagi@nagasaki-u.ac.jp 
to $50 \%$ of patients with adenocarcinoma of the lung, pancreas, or breast, and in $10 \%$ or less of patients with gastric, colon, or rectal cancers (Kohno et al. 1992). On the other hand, despite the expression of MUC1 and its ligand, L-selectin, in normal and malignant hematopoietic cells, the information is limited about the clinical significance of soluble KL-6 in patients with hematological malignancies (Brossart et al. 2001; Mukherjee et al. 2005). Moreover, hematological malignancies are frequently accompanied by interstitial pneumonia related to infectious agents, therapeutic drugs, and radiation and the possible relationship of KL-6 to these complications is unknown.

The protein SP-D, a member of the human collectin family, is produced mainly in the lung by alveolar type II and bronchiolar epithelial cells. The serum level of SP-D is considered to reflect damage from, or response of epithelial cells to, inflammation (Leth-Larsen et al. 2003). Accordingly, SP-D is also a useful marker for interstitial pneumonia as well as for infectious pulmonary diseases. These facts prompted us to study the serum levels of KL-6 and SP-D in patients with hematological malignancies. In particular, we focused on serum KL-6 status in adult T-cell leukemia (ATL), because ATL is characterized by a high number of invasive tumor cells and by complications due to infective interstitial pneumonias related to non-bacterial pathogens (White et al. 1995). To understand the pathological and clinical implications of serum KL-6 and SP-D, the potential correlation between their levels and those of other serum biomarkers such as lactate dehydrogenase (LD) activity, and soluble IL-2 receptor (sIL-2R) levels were examined.

Here we present the finding that serum KL-6 and SP-D are elevated in a small fraction of patients with hematological malignancies. Their elevation in serum is mutually exclusive and the measurement of KL-6 and SP-D is helpful to discriminate leukemic invasion into the lung from infectious pulmonary complications in ATL.

\section{Patients and Materials}

Serum samples from patients and healthy volunteers were collected after informed consent based on the study protocol approved the Ethics Committee of Nagasaki University Hospital (Nagasaki, Japan). A total of 363 serum samples were collected at the time of diagnosis and relapse, and stored at $-30^{\circ} \mathrm{C}$ until use. We examined the serum levels of KL-6 and SP-D in sera from 128 patients $(\mathrm{M}: \mathrm{F}=$ 76 : 52, Range: 4-84 years, Mean: 59) with hematological malignancies. The patients with hematological malignancies consisted of patients with adult T-cell leukemia (ATL; 67 patients), acute lymphoblastic leukemias (ALL; 9 patients), acute myeloid leukemia (AML; 9 patients), B-cell malignant lymphoma (ML; 14 patients), chronic lymphocytic leukemias (CLL; 12 patients), chromic myeloid leukemia (CML; 6 patients), and HTLV-1 carriers with other diseases (11 patients). All of these diseases were diagnosed based on routine clinico-pathological findings, such as morphology, immunophenotype, and cyto/molecular genetics. ATL was subclassified into 4 categories of acute, lymphoma, chronic, and smoldering subtypes according to LSG criteria (Shimoyama 1991).

\section{Methods}

\section{$K L-6$ and SP-D quantification:}

Serum KL-6 was quantified using the PICOLUMI KL-6 kit (Sanko Junyaku, Tokyo, Japan), an electrochemiluminescent immunoassay (ECLIA) method specific for human KL-6, according to the manufacturer's instructions. Briefly, $200 \mu \mathrm{l}$ serum sample, pre-diluted $1: 51$ with the sample diluent, was incubated with $25 \mu 1$ anti-KL-6 monoclonal antibody-coated micro magnetic beads solution for $9 \mathrm{~min}$ at $30^{\circ} \mathrm{C}$. After the beads were washed twice with the washing solution, $200 \mu 1$ ruthenium (Ru)-labeled anti-KL-6 monoclonal antibody was added to the beads and incubated for $9 \mathrm{~min}$ at $30^{\circ} \mathrm{C}$. After the beads were washed three times with the washing solution, the beads were placed into the electrode and the photons (wavelength, $620 \mathrm{~nm}$ ) emitted from the Ru-labeled anti-KL-6 monoclonal antibody were counted with a photo-multiplier tube. The concentration of KL-6 in the serum sample was calculated by comparing the obtained counts with photon counts obtained from calibrated KL-6 standard antigens. These ECLIA procedures were carried out with an automatic ECLIA analyzer (PICOLUMI 8220; Sanko Junyaku, Tokyo, Japan).

Serum SP-D was quantified with an SP-D EIA kit (Yamasa Co., Chiba, Japan) according to the manufacturer's instructions. Briefly, $100 \mu 1$ serum sample, pre-diluted 1:11 with the sample diluent, was put into a 96-well microplate coated with an anti-SP-D monoclonal antibody. The serum sample was incubated for $18-24 \mathrm{~h}$ at $2-8^{\circ} \mathrm{C}$. After the well was washed three times with washing solution, $100 \mu 1$ monoclonal antibody-labeled peroxidase enzyme was added and incubated for $2 \mathrm{~h}$ at $15-30^{\circ} \mathrm{C}$. After the well was washed three times with washing solution, $100 \mu 1$ color development reagent including tetramethylbenzidine and hydrogen peroxide was added and incubated for $15 \mathrm{~min}$ at $15-30^{\circ} \mathrm{C}$ followed by addition of $100 \mu \mathrm{l}$ stop solution. The absorbance of the solution in the well was measured in a microplate reader at a wavelength of $450 \mathrm{~nm}$. The concentration of SP-D in the serum sample was calculated by comparing the obtained absorbance with the absorbance of calibrated SP-D standard antigens.

In addition, to evaluate the tumor behavior of ATL, soluble interleukin-2 receptor (sIL-2R) was measured according to a previously described method (Kamihira et al. 1994).

\section{KL-6 and SP-D expression in ATL cells:}

The subcellular expression of KL-6 and SP-D was examined by flow cytometry and indirect immunostaining using anti-SP-D (Yamasa, Tokyo, Japan) and anti-KL-6 (Eisai, Tokyo, Japan) antibodies and an FITC-conjugated goat anti-mouse antibody (Dako, Kyoto, Japan). The fluorescent signals were evaluated using FACSCalibur and Cellquest software (BD Biosciences Immunocytometry System, San Jose, CA) and fluorescence microscopy.

\section{Statistical analysis}

Results are expressed as mean \pm standard deviation (s.D.) unless mentioned. Unpaired data were analyzed by Wilcoxon's rank sum test and Mann-Whitney $U$ test, respectively. Ratio was evaluated utilizing Fisher's exact probability test. Correlation was evaluated by Spearman's correlation index. A two-tailed $p$ value of 0.05 was considered significant. 


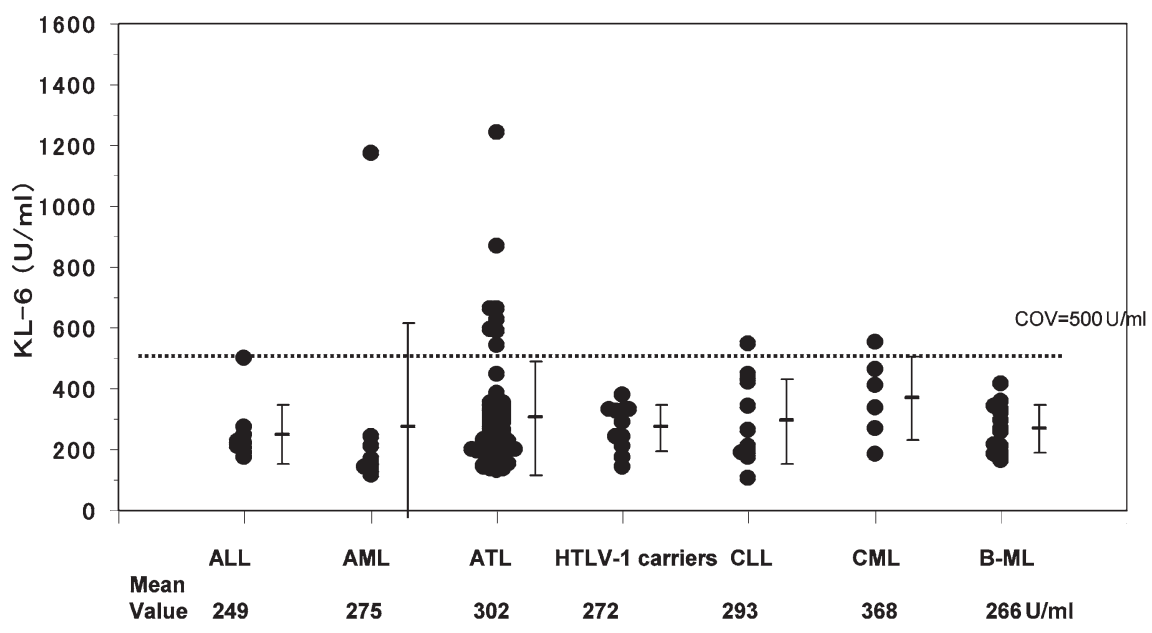

Fig. 1. Distribution plots of the serum level of KL-6 among each disease category in 128 patients with hematological malignancies. The serum level of KL-6 was higher than the cut off value of $500 \mathrm{U} / \mathrm{ml}$ (dotted line) in only 11 out of 128 patients with hematological malignancies. No statistically significant difference in the incidence or the median level among disease categories was observed.

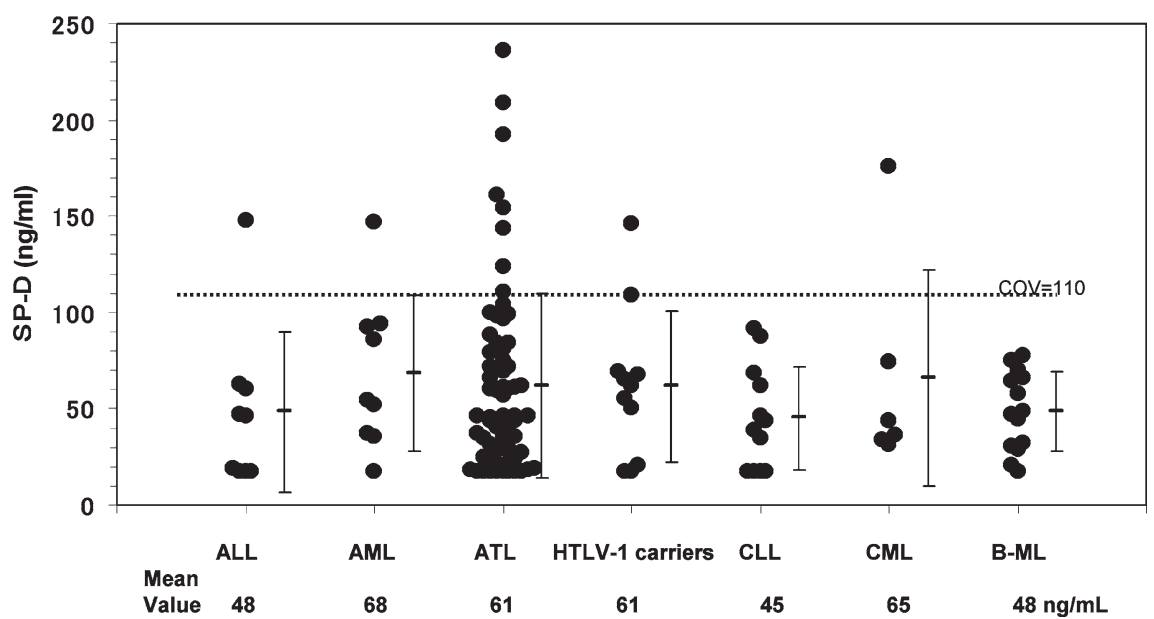

Fig. 2. Distribution plots of serum SP-D among disease categories. Serum SP-D was measured in the same 128 patients as in Fig. 1 using a cut-off point of $110 \mathrm{ng} / \mathrm{ml}$ (dotted line). The distribution of the plots is similar to that of Fig. 1.

\section{Results}

KL-6 and SP-D levels in sera from patients with hematological malignancies

Serum KL-6 and SP-D levels were measured in 128 serum samples from patients with various hematological malignancies, as shown in Figs. 1 and 2, respectively. The cut-off value was set at $500 \mathrm{U} / \mathrm{ml}$ for KL-6 and $110 \mathrm{ng} / \mathrm{ml}$ for SP-D, based on the data from healthy volunteers. Levels of KL-6 that were elevated above the cut-off value were detected in only $11(8.9 \%)$ of 128 cases and these cases consisted of $1(11.1 \%)$ of 9 AML, $8(11.9 \%)$ of 67 ATL, $1(8.3 \%)$ of 12 CLL, and $1(16.7 \%)$ of 6 CML. No statistically significant difference in the mean value was found among the disease groups whose mean values ranged from 249 to $368 \mathrm{U} / \mathrm{ml}$. The frequency of high serum SP-D levels in each disease group was similar to that of serum KL-6: $11 \%(1 / 9)$ in ALL, $11 \%(1 / 9)$ in AML, $12 \%(8 / 67)$ in ATL,
$18 \%(2 / 11)$ in HTLV-1 carriers, $0 \%(0 / 12)$ in CLL, $17 \%$ $(1 / 6)$ in CML, and $0 \%(0 / 14)$ in ML. The overall frequency is $10.2 \%$ (13/128 cases).

We next examined the potential mutual relationship between elevated levels of KL-6 and SP-D in ATL patients (Fig. 3), but found no statistically significant correlation $(r$ $=0.1096, p=0.218$ ). Interestingly, the elevation in the serum levels of KL-6 or SP-D was almost completely mutually exclusive. Thus, only 2 patients showed elevated levels of both markers, while KL-6 was exclusively elevated in 11 patients and SP-D was exclusively elevated in 10 patients.

\section{KL-6 and SP-D are elevated in a mutually exclusive manner in ATL}

To determine the clinical significance of the difference in expression levels of KL-6 and SP-D and their potential use as biomarkers, further investigations were carried out using samples from ATL patients. Fifteen out of 67 ATL 


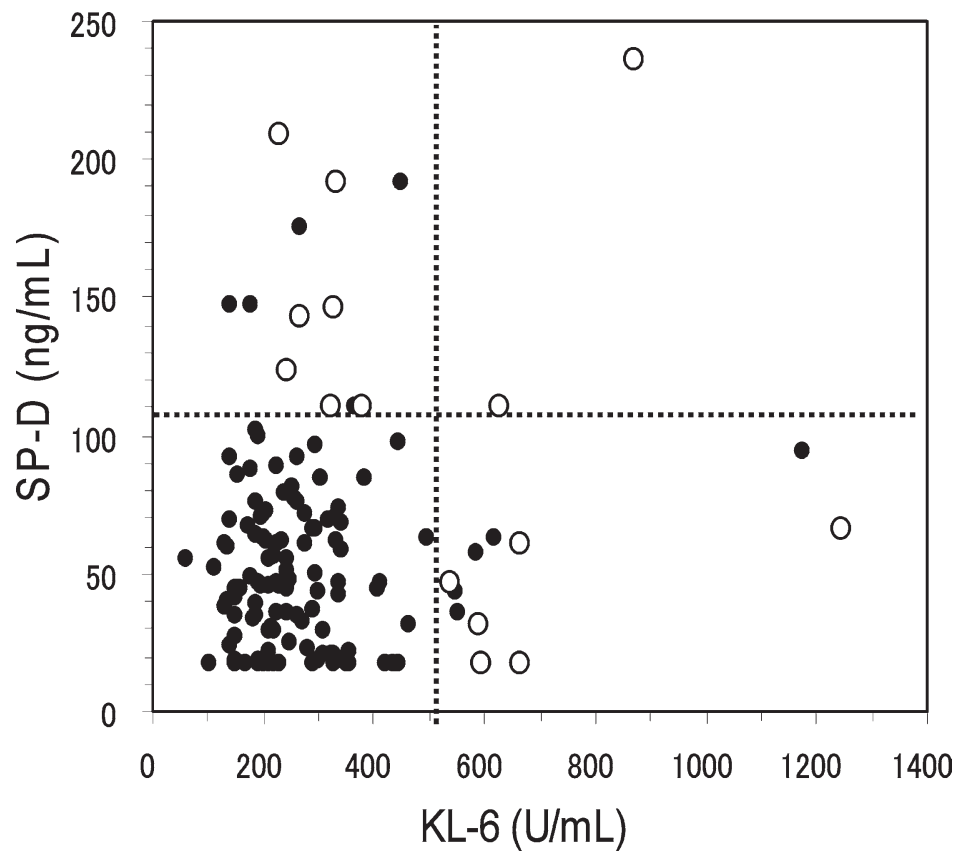

Fig. 3. Analysis of the potential relationship between serum KL-6 and SP-D levels in patients with hematological malignancies. A twin dot plot graph between KL-6 and SP-D showed no correlation between their expression levels $(\mathrm{y}=0.028 \mathrm{x}$ $+50.6, r=0.1096, p=0.218$ ). Both markers were elevated in only 2 patients. KL-6 was exclusively elevated in 11 patients, and SP-D was exclusively elevated in 10 patients. Open circles represent ATL patients with elevated levels of KL-6 or SP-D. Closed circles represent all other patients with hematological malignancies, including ATL patients.

Table 1. The clinico-pathological features of 15 patients with elevated levels of either KL-6 or SP-D.

\begin{tabular}{|c|c|c|c|c|c|c|c|c|}
\hline \multirow{2}{*}{$\begin{array}{l}\text { Patient } \\
\text { No. }\end{array}$} & \multirow{2}{*}{ Age } & \multirow{2}{*}{ Gender } & \multirow{2}{*}{ Subtype of ATL } & \multirow{2}{*}{$\begin{array}{c}\mathrm{KL}-6 \\
(\mathrm{U} / \mathrm{mL})\end{array}$} & \multirow{2}{*}{$\begin{array}{c}\text { SP-D } \\
(\mathrm{ng} / \mathrm{mL})\end{array}$} & \multicolumn{3}{|c|}{ Pulmonary complications } \\
\hline & & & & & & & Tumorous & Infectious \\
\hline 1 & 76 & $\mathrm{~F}$ & Lymphoma/acute & 628 & 110.3 & $\pm^{*}$ & KL-6 expressing ATL cells ${ }^{1)}$ & - \\
\hline 2 & 56 & $\mathrm{~F}$ & Chronic & 871 & 235.8 & + & Leukemic infiltration $^{2)}$ & + \\
\hline 3 & 64 & M & Chronic & 1244 & 66.2 & - & KL-6 expressing ATL cells ${ }^{1}$ & - \\
\hline 4 & 81 & M & Acute & 665 & 17.2 & + & Leukemic infiltration $^{2)} \& \mathrm{PE}$ & - \\
\hline 5 & 70 & M & Lymphoma & 665 & 60.6 & & $\pm^{*}$ & - \\
\hline 6 & 78 & M & Lymphoma & 540 & 46.2 & & $\pm^{*}$ & - \\
\hline 7 & 55 & M & Lymphoma & 595 & 17.1 & & $\pm *$ & - \\
\hline 8 & 73 & $\mathrm{~F}$ & Smoldering & 592 & 31.1 & & - & - \\
\hline 9 & 73 & M & Smoldering & 380 & 110.1 & & - & + \\
\hline 10 & 72 & M & Lymphoma & 331 & 192.1 & & - & + \\
\hline 11 & 35 & M & Smoldering & 327 & 146.4 & & - & + \\
\hline 12 & 40 & M & Chronic & 322 & 110.1 & & - & + \\
\hline 13 & 82 & M & Smoldering & 268 & 143.2 & & - & + \\
\hline 14 & 43 & $\mathrm{~F}$ & Acute & 244 & 123.4 & & - & + \\
\hline 15 & 40 & $\mathrm{~F}$ & Acute & 230 & 209.1 & & - & + \\
\hline
\end{tabular}

The group comprising patients 1-8 showed elevated KL-6 and was characterized by no evidence of overt pulmonary infectious complications, but had demonstrable leukemic cell invasion into the lungs (patients 2-5) and disseminated tumor cells in the body (patients 6-7). In contrast, patients (numbers 9-15) with exclusive elevation of SP-D had only infectious pulmonary complications. The cut-off values used were $500 \mathrm{U} / \mathrm{ml}$ for KL-6 and $110 \mathrm{ng} / \mathrm{ml}$ for SP-D.

${ }^{1)}$ KL-6-bearing ATL cells; ${ }^{2)}$ demonstrated by BALF cytology. ${ }^{3)}$ ATL, AML, and gastric cancer

*Clinically suspected with reticular shadow on Chest X-p,

PE, pleural effusion; CBP, chronic broncho-pneumonia; BP, broncho-pneumonia; CMV pn, cytomegalovirus pneumonia. 
a) Flow cytometric analysis of KL- 6 expression in patients 1 and 3
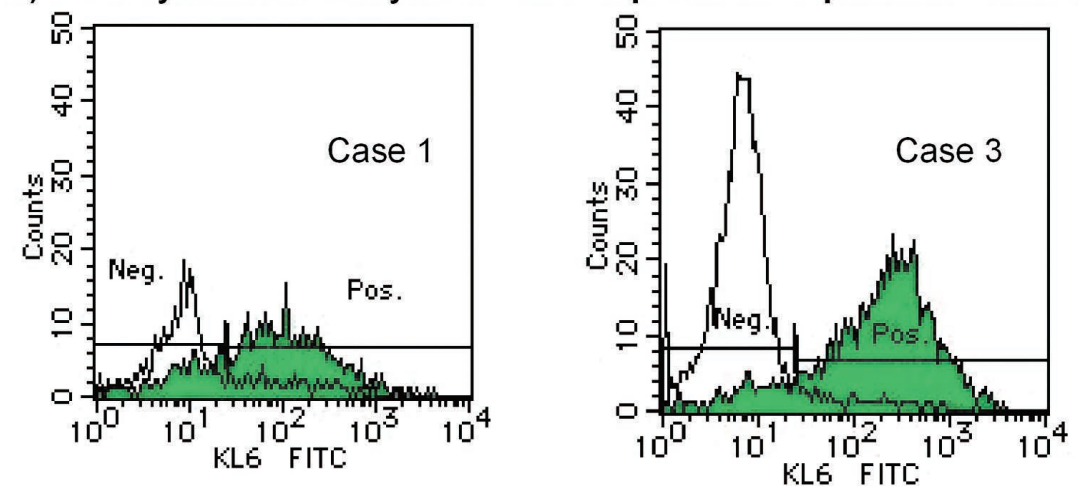

b) Immuno fluorescence analysis of KL-6 and SP-D in patients 1 and 3
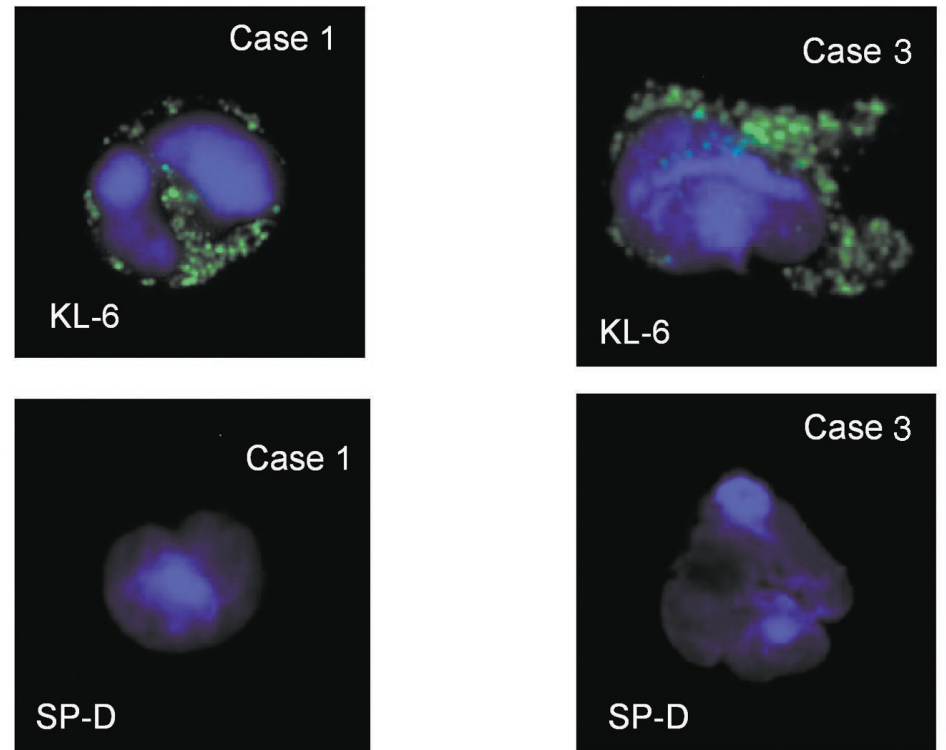

Fig. 4. Subcellular expression of KL-6 and SP-D in ATL cells. The subcellular expression of KL-6 and SP-D was examined by flow cytometry (a) and indirect immunostaining using anti-SP-D and anti-KL-6 antibodies (b). (a) Flow cytometric analysis revealed positive histograms (green) for KL-6, but negative for SP-D (neg., white) in ATL cells of patients 1 and 3. These patient numbers are consistent with Table 1. (b) Immunofluorescence staining showed positive signals (green) only for KL-6, and not for SP-D, in a smear of cells with multi-lobulated nuclei. The blue stains are nuclei labeled with DAPI.

patients had the elevated level of either KL-6 or SP-D, as shown in Table 1. Both markers were elevated in 2 patients (numbers 1 and 2). KL-6 was exclusively elevated in 6 patients (numbers 3 to 8), and SP-D was exclusively elevated in 7 patients (numbers 9 to 15). Interestingly, there was a substantial difference depending on the type of pulmonary complications observed in the groups exclusively expressing KL-6 or Sp-D (Table 1). Thus, the KL-6-expressing group was characterized by a predominance of lymphoma subtype (4 patients), leukemic cell invasion into the lungs (patients 2 and 4), demonstrated by cytology using bronchialveolar lavage fluid (BALF) or clinically suspected due to a reticular shadow on chest X-p (numbers 5, 6, and 7). In contrast, patients with a high serum level of only SP-D had pulmonary infectious complications but no leukemia-related pulmonary lesions.

To better understand the origin of serum KL-6 and
SP-D, their subcellular expression was examined in ATL cells from 20 patients by flow cytometry and indirect immunostaining. KL-6 was detected with variable intensity on the cell surface as well as in the cytoplasm in 10 of the 20 ATL patients tested, whereas SP-D was not detectable in any patients. In particular, ATL cells obtained from patients 1 and 3 abundantly expressed KL-6, but not SP-D (Fig. 4 a and $b$ ). Hyperplasia of type II pneumocytes was histologically observed in a patient with ATL with pulmonary leukemic invasion (Fig. 5). All of these data suggest that KL-6 in the serum is partially derived from these pneumocytes or from the ATL cells themselves.

We then compared KL-6 and SP-D serum levels with LD activity and sIL-2R levels that are well-characterized biomarkers. sIL-2R is a good surrogate marker for the evaluation of malignant behavior and tumor burden in ATL. There was neither a statistically significant correlation 


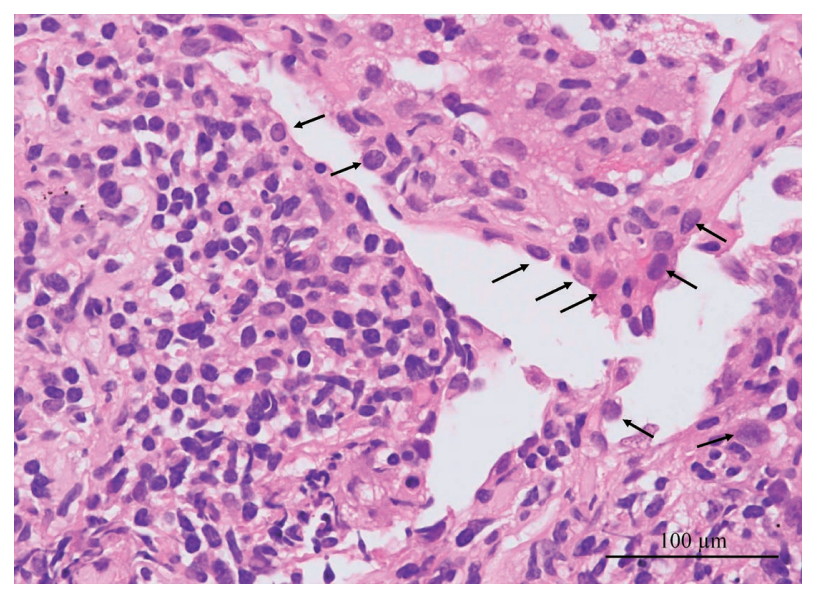

Fig. 5. Histological examination of a lung sample with ATL cell invasion. Hyperplasia (arrow head) of type II pneumocytes was observed with massive invasion of CD4positive ATL cells (Patient No.4 in Table1). Hyperplasia of type II pneumocytes indicated pulmonary leukemic invasion of ATL cells.

between KL-6 and LD $(r=-0.012, p=0.959)$, nor between KL-6 and sIL-2R ( $r=0.0126, p=0.919)$, suggesting that the clinical significance of KL-6 is independent of the pathology represented by LD or sIL-2R. In contrast, SP-D had a tendency to correlate with LD but not with sIL-2R, further suggesting that the clinical significance of KL-6 and SP-D differs.

\section{Discussion}

We present the data that show an elevation of serum KL-6 and SP-D levels in about $10 \%$ of patients with hematological malignancies. Interestingly, the elevation of these markers was mutually exclusive. There was no noticeable difference in the incidence of the elevated serum KL-6 and SP-D among disease subtypes of ALL, AML, CML, CLL, ML, and ATL. The only reports we found in the literature were several case reports showing that serum KL-6 happened to be elevated when cytomegalovirus or pneumocystis jiveroci pneumonia was present as a complication during intensive chemotherapy in patients with ALL (Takahashi et al. 2001), ML and ATL (Tanaka et al. 2002). More interestingly, Fujisawa et al. (2007) reported a case of peripheral T cell lymphoma (PTCL) with elevated serum KL-6 derived from reactive proliferative pneumocytes due to pulmonary invasion of tumor cells. These data indicate that soluble KL-6 in the serum is associated with pulmonary complications of infective interstitial pneumonia rather than hematological malignancies. However, our data presented here differ from these previous reports in that patients with high serum levels of KL-6 showed a trend of leukemic infiltration into the lung, but no overt infectious pulmonary complications, as shown for the ATL patients in Table 1. On the other hand, SP-D may be a useful marker of the presence of infectious lung diseases that occur as a complication in patients with ATL. Indeed, none of 7 patients that showed exclusive elevation of SP-D had leukemic cell invasion into the lung, but all of these patients did have complications with infectious pulmonary disorders. All of these findings suggest that measurement of the combined levels of serum KL-6 and SP-D represents a possible method to discriminate leukemic invasion from infectious pulmonary complications, at least in ATL. At present, there are difficulties in the invasive collection of BALF for differential diagnosis of leukemic invasion or interstitial pneumonias, which are frequent complications in ATL. Therefore, KL-6 and SP-D are promising markers for such differential diagnosis, because the assay methods are simple and are measured without invasion.

Currently, the origin of serum KL-6 in cancer patients is controversial. Although there are reports that cancer cells themselves produce KL-6 (Kohno et al. 1992), our data indicate that ATL cells probably also express KL-6, suggesting that serum KL-6 originates from ATL cells. However, elevated serum KL-6 in ATL was observed only in patients with pulmonary leukemic cell invasion, but not in ATL patients without this complication. In addition, hyperplasia of type II pneumocytes was histologically observed in an ATL patient with leukemic lung invasion. These findings suggest that a biological relationship exists between pneumocytes and ATL cells via KL-6 with regard to lung invasion and cell-growth at invasive sites. That is, aberrant overexpression of KL-6 may account in part for the lung invasion of ATL cells, because KL- 6 has been recently shown to be involved in cell-cell co-adhesive infiltration and proliferation (Teruya-Feldstein et al. 2003).

In conclusion, although serum KL-6 and SP-D are rarely elevated in patients with hematological malignancies including ATL, the elevation of either KL-6 or SP-D can be used as a marker to discriminate leukemic invasion from infectious pulmonary complications.

\section{References}

Brossart, P., Schneider, A., Dill, P., Schammann, T., Grunebach, F., Wirths, S., Kanz, L., Bühring, H.J. \& Brugger, W. (2001) The epithelial tumor antigen MUC1 is expressed in hematological malignancies and is recognizaed by MUC1-specific cytotoxic T-lymphocytes. Cancer Res., 61, 6846-6850.

Fujisawa, T., Suda, T., Matsuura, S., Enomoto, N., Takeshita, K., Ohnishi, K. \& Chida, K. (2007) Peripheral T-cell lymphoma with diffuse pulmonary infiltration and an increase in serum KL-6 level. Respirology, 12, 452-454.

Kamihira, S., Atogami, S., Sohda, H., Momita, S., Yamada, Y. \& Tomonaga, M. (1994) Significance of soluble interleukin-2 receptor levels for evaluation of the progression of adult T-cell leukemia. Cancer, 73, 2753-2758.

Kohno, N. (1999) Serum marker KL-6/MUC1 for the diagnosis and management of interstitial pneumonitis. J. Med. Invest, 46, $151-158$.

Kohno, N., Akiyama, M., Kyoizumi, S., Hakoda, M., Kobuke, K. \& Yamakido, M. (1988) Detection of soluble tumor-associated antigens in sera and effusions using novel monoclonal antibodies, KL-3 and KL-6, against lung adenocarcinoma. Jpn. J. Clin. Oncol., 18, 203-216.

Kohno, N., Hamada, H., Fujioka, S., Hiwada, K., Yamakido, M. \& Akiyama, N. (1992) Circulating antigen KL-6 and lactate 
dehydrogenase for monitoring irradiated patients with lung cancer. Chest, 102, 117-122.

Leth-Larsen, R., Nordenbaek, C., Tornoe, I., Moller, V., Schlosser, A., Koch, C., Teisner, B., Junker, P. \& Holmskov, U. (2003) SP-D serum levels in patients with community-acquired small star, filled. Clin. Immunol., 108, 29-37.

Marcaurelle, L.A. \& Bertozzi, C.R. (2002) Recent advances in the chemical synthesis of mucin-like glycoproteins. Glycobiology, 12, 69R-77R.

Mukherjee, P., Tinder, T.L., Basu, G.D. \& Gendler, S.J. (2005) MUC1(CD227) interacts with lck tyrosine kinase in Jurkat lymphoma cells and normal T cells. J. Leukoc. Biol., 77, 90-99.

Shimoyama, M. (1991) Diagnostic criteria and classification of clinical subtypes of adult T-cell leukaemia-lymphoma. A report from the Lymphoma Study Group (1984-87). Br. J. Haematol., 79, 428-437.

Stahel, R.A., Gilks, W.R., Lehmann, H.P. \& Schenker, T. (1994) Third international workshop on Lung Tumor and differentiation antigens: overview of the results of the central data analy- sis. Int. J. Cancer, Suppl, 8, 6-26.

Takahashi, T., Ebihara, Y., Manabe, A., Tsuji, K., Nakamura, T., Nakahata, T. \& Iwamoto, A. (2001) SP-D and KL-6 as a serologic indicators of pneumocystis carinii pneumonia in a child with acute lymphoblastic leukemia. J. Med., 32, 41-51.

Tanaka, M., Tanaka, K., Fukahori, S., Fujimatsu, Y., Jojima, H., Shiraishi, K., Honda, J. \& Oizumi, K. (2002) Elevation of serum KL-6 levels in patients with hematological malignancies associated with cytomegalovirus or Pneumocysis carinii pneumonia. Hematology, 7, 105-108.

Teruya-Feldstein, J., Donnelly, G.B., Goy, A., Hegde, A., Nanjangud, G., Qin, J., Thaler, H., Gilles, F., Dyomin, V.G., Lloyd, K.O., Zelenetz, A.D., Houldsworth, J. \& Chaganti, R.S. (2003) MUC-1 mucin protein expression in B-cell lymphomas. Appl. Immunohistochem. Mol. Morphol., 11, 28-32.

White, J.D., Zaknoen, S.L., Kasten-Sportes, C., Top, L.E., Navarro-Roman, L., Nelson, D.L. \& Waldmann, T.A. (1995) Infectious complications and immunodeficiency in patients with human T-cell lymphotropic virus I-associated adult T-cell leukemia/lymphoma. Cancer, 75, 1598-1607. 